\title{
1 \\ Definition, diagnosis, and origin of extreme weather and climate events
}

\author{
DAVID B. STEPHENSON
}

\section{Condensed summary}

Extreme weather and climate events are a major source of risk for all human societies. There is a pressing need for more research on such events. Various societal changes, such as increased populations in coastal and urban areas and increasingly complex infrastructure, have made us potentially more vulnerable to such events than we were in the past. In addition, the properties of extreme weather and climate events are likely to change in the twenty-first century owing to anthropogenic climate change.

The definition, classification, and diagnosis of extreme events are far from simple. There is no universal unique definition of what is an extreme event. This chapter discusses these issues and presents a simple framework for understanding extreme events that will help enable future work in this important area of climate science and global reinsurance.

\subsection{Introduction}

Human society is particularly vulnerable to severe weather and climate events that cause damage to property and infrastructure, injury, and even loss of life. Although generally rare at any particular location, such events cause a disproportionate amount of loss.

In this chapter, I attempt to describe a framework for classifying, diagnosing, and understanding extreme weather and climate events. The multidimensional aspect of complex extreme events will be discussed, and statistical methods will be presented for understanding simple extreme events. Some preliminary ideas about the origin of extreme events will also be presented. 


\subsection{Definition of extreme events}

Extreme events are generally easy to recognize but difficult to define. This is due to several reasons. First, there is no unique definition for what is meant by the word "extreme": several definitions are in common use. Second, the concept of "extremeness" is relative and so strongly depends on context. Third, the words "severe," "rare," "extreme," and "high-impact" are often used interchangeably.

\subsubsection{Severe, rare, extreme, or high-impact?}

In an attempt to alleviate some of the confusion, here are some definitions of these terms.

- Severe events are events that create large losses in measures such as number of lives, financial capital, or environmental quality (e.g., loss of species). The severity can be measured by the expected long-term loss, which is known as the risk. Risk depends on the product of the probability of the event (the hazard), the exposure to the hazards (e.g., how many people are exposed), and the vulnerability (i.e., how much damage ensues when someone is hit by the event). In other words, severity is a function of not only the meteorological hazard but also the human state of affairs. For example, the severity of US landfall hurricanes has increased considerably in recent years, mainly owing to increased numbers of people settling in the US Gulf states (increased exposure).

- Rare events are events that have a low probability of occurrence. Because of the rarity of these events, human societies (and other ecosystems) are often not well adapted to them and so suffer large amounts of damage when they do occur. Hence, despite their rarity, the large vulnerability associated with such events can often lead to large mean losses (and hence they are a type of severe event).

- Extreme events are events that have extreme values of certain important meteorological variables. Damage is often caused by extreme values of certain meteorological variables, such as large amounts of precipitation (e.g., floods), high wind speeds (e.g., cyclones), high temperatures (e.g., heat waves), etc. Extreme is generally defined as either taking maximum values or exceedance above pre-existing high thresholds. Such events are generally rare; for example, extreme wind speeds exceeding the 100-year return value, which have a probability of only 0.01 of occurring in any particular year.

- High-impact events are severe events that can be either short-lived weather systems (e.g., severe storms) or longer-duration events such as blocking episodes that can lead to prolonged heat waves and droughts. The World Meteorological Organization (WMO) program THORPEX uses the phrase "high-impact weather" rather than "severe weather" to help people avoid confusing the term severe with only short-lived events such as individual storms (D. Burridge, personal communication). 


\subsubsection{Multidimensional nature of extreme events}

In addition to this potential source of confusion, extreme events have a variety of different attributes and so cannot be completely described by a single number. The multidimensional nature of extreme events is often overlooked in rankings of the events based on only one of the attributes (e.g., the category numbers for hurricanes based solely on maximum surface wind speed).

Extreme events have attributes such as:

- rate (probability per unit time) of occurrence

- magnitude (intensity)

- temporal duration and timing

- spatial scale (footprint)

- multivariate dependencies

For example, a major hurricane is rare, has large-magnitude surface wind speeds, has a generally large spatial scale (with the exception of certain smallspatial-footprint events such as Hurricane Camille in 1969), and develops over synoptic timescales ranging from hours to several days. In addition, the severity of such events can also depend on the combination of extreme behavior in more than one variable; for example, much hurricane damage is due to extreme precipitation as well as extreme wind speeds. For example, a severe ice storm can involve conditions for all three variables: temperature, wind, and precipitation amount. The Intergovernmental Panel on Climate Change (IPCC, 2001) defines "complex extreme" events as "severe weather associated with particular climatic phenomena, often requiring a critical combination of variables." The magnitude of the losses depends on several meteorological variables and hence dependencies between them.

The temporal duration of extreme events plays an important role in the exposure and hence total losses. For example, the long duration of the flooding in New Orleans caused by Hurricane Katrina led to large insurance losses due to business interruption in addition to property damage losses. Temporal duration also provides a useful way of classifying extreme events. The duration is implicit when one describes an event as a "climate" extreme event rather than a "weather" extreme event. The medical illness concepts of chronic and acute can be usefully applied to weather and climate events:

- Acute extremes: events that have a rapid onset and follow a short but severe course.

Examples are short-lived weather systems such as tropical and extratropical cyclones, polar lows, and convective storms with extreme values of meteorological variables such as wind speed and precipitation that can lead to devastating wind, 
flood, and ice damage. In addition to these obvious examples of high-impact extreme events, there are less obvious acute extreme events, such as fog that causes major transport disruption (e.g., at airports).

- Chronic extremes: events that last for a long period of time (e.g., longer than 3 months) or are marked by frequent recurrence. Examples are heat waves and droughts that can lead to such impacts as critical water shortages, crop failure, heat-related illness and mortality, and agricultural failure. Because of their extended duration and generally lower intensity, chronic extreme events can often be harder to define than acute extreme events, but they have the advantage that there is more time to issue warnings and take protective actions. Note that not all high-impact weather events are acute; for example, blocking weather events that last several days are chronic events.

\subsubsection{A simple taxonomy}

One approach towards creating a definition is to try to list and classify all events that one considers to be extreme. For example, the following events are often cited as examples of extreme weather/climate events.

- Tropical cyclones and hurricanes (e.g., Typhoon Tracy, Hurricane Hugo, etc.). These storms are the major source of global insured catastrophe loss after earthquakes.

- Extratropical cyclones (e.g., the "Perfect Storm" that hit the northeast coast of the United States, October 28-30, 1991). These storms are generally referred to as "windstorms" by the reinsurance industry.

- Convective phenomena such as tornadoes, waterspouts, and severe thunderstorms. These phenomena can lead to extreme local wind speeds and precipitation amounts on horizontal scales of up to about $10 \mathrm{~km}$. Deep convection often leads to precipitation in the form of hail, which can be very damaging to crops, cars, and property.

- Mesoscale phenomena such as polar lows, mesoscale convective systems, and sting jets. These features can lead to extreme wind speeds and precipitation amounts on horizontal scales from 100 to $1,000 \mathrm{~km}$.

- Floods of rivers, lakes, coasts, etc., due to severe weather conditions; for example, river floods caused by intense precipitation over a short period (e.g., flash floods) and persistent/recurrent precipitation over many days (e.g., wintertime floods in northern Europe), river floods caused by rapid snowmelt due to a sudden warm spell, or coastal floods caused by high sea levels due to wind-related storm surges.

- Drought. Meteorological drought is defined usually on the basis of the degree of dryness (in comparison to some "normal" or average amount) and the duration of the dry period. Simple definitions relate actual precipitation departures to average amounts on monthly, seasonal, or annual timescales. However, meteorological 


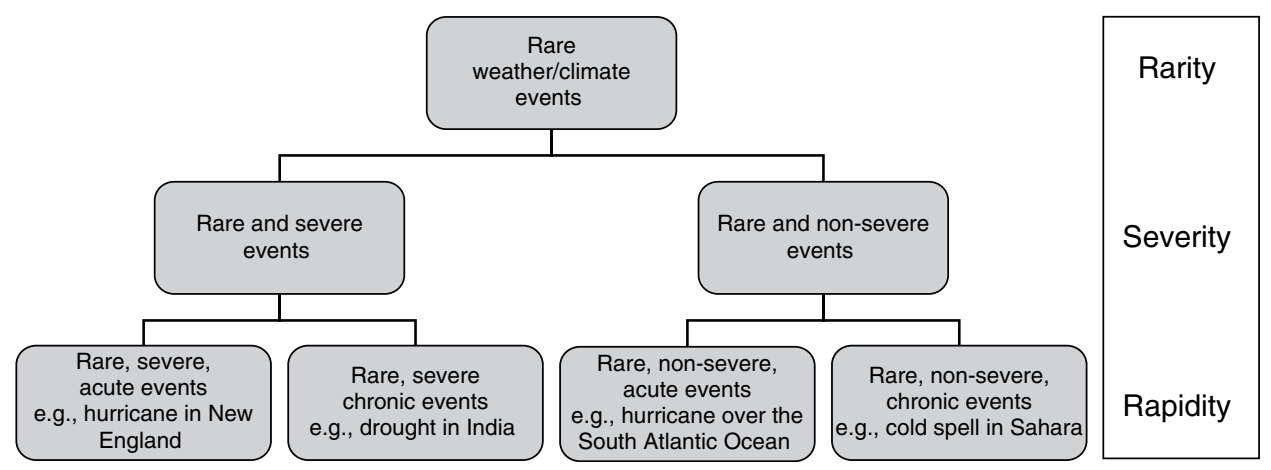

Figure 1.1. Simple taxonomy of extreme weather and climate events.

drought also depends on other quantities such as evaporation that depend on variables such as temperature.

- Heat waves. Periods of exceptionally warm temperatures can have profound impacts on human health and agriculture. Duration is a key component determining the impact.

- Cold waves/spells (e.g., extremely cold days or a succession of frost days with minimum temperatures below $0{ }^{\circ} \mathrm{C}$ ).

- Fog. Extremely low visibility has major impacts on various sectors such as aviation and road transport.

In order to make more sense of this set of diverse events, it is useful to try to classify them into smaller subgroups. A simple binary taxonomy of weather and climate events can be based on the attributes of rarity, severity, and duration as shown in Figure 1.1.

First, for any particular location an event can be considered to be either rare or not rare depending on how often such an event happens. For example, events can be considered rare if they happen less frequently than once every 250 years (a return period often used by the reinsurance industry to assess acceptable levels of risk).

Second, depending upon its impacts, an event can be considered either severe or not severe. Severity depends not only upon the characteristics of an event but also upon the exposure and vulnerability of the system it impacts. For example, a heat wave in the Gobi Desert is not a severe event in terms of human impact, because there is very little human exposure (i.e., very few people live there).

Finally, events can be classified by their longevity into either acute or chronic events. In contrast to the medical situation, where severe syndromes can generally not be sustained over a long period of time and therefore tend to be acute rather than chronic (e.g., severe acute respiratory syndrome [SARS]), 
severe weather events can be either acute (e.g., a major hurricane) or chronic (e.g., a major drought).

\subsection{Statistical diagnosis of extreme events}

This section will briefly describe some statistical approaches for interpreting extreme events. A more comprehensive discussion is given in the excellent book by Coles (2001).

\subsubsection{Point process modeling of simple extreme events}

In order to make the analysis more amenable to mathematical modeling, it is useful to neglect (important!) attributes such as temporal duration, spatial scale, and multivariate dependencies. The IPCC (2001) defined "simple extreme" events to be "individual local weather variables exceeding critical levels on a continuous scale."

This highly simplified view of a complex extreme event is widely used in weather and climate research. However, one can always consider an event as a simple extreme in overall loss (i.e., severity) no matter how complex the underlying meteorological situation may be.

Simple extreme events, defined as having exceedances above a high threshold, are amenable to various types of statistical analysis. Because exceedances occur at irregular times and the excesses tend to be strongly skewed, such series are not amenable to the usual methods of time series analysis. However, exceedances can be considered to be a realization of a stochastic process known as a marked point process: a process with random magnitude marks (the excesses above the threshold) that occurs at random points in time (see Diggle, 1983; Cox and Isham, 2000). Rare exceedances above a sufficiently high threshold can be described by a nonhomogenous Poisson process (Coles, 2001).

Point process methods have been widely used in various areas of science; for example, in providing a framework for earthquake risk assessment and prediction in seismology (Daley and Vere-Jones, 2002). Point process methods can be used to explore and summarize such records and are invaluable for making inferences about the underlying process that gave rise to the record. Broadly speaking, this analysis is performed by considering statistical properties of the points, such as the number of events expected to occur per unit time interval (the rate/intensity of the process), statistical properties of the marks (the probability distribution of the excesses), and joint properties such as how the marks depend on the position and spacing of the points, the magnitude of preceding events, etc. 


\subsubsection{Example: central England temperature observations}

Typical time series resemble the example of 3,080 monthly means of observed central England temperature (CET) shown in Figure 1.2.

The 308 warm extreme events with temperatures in excess of the 90th quantile of $15.6{ }^{\circ} \mathrm{C}$ (the dots above the solid line in the upper panel) form a realization of a marked point process. Rather than being recorded continuously, this example and many other meteorological variables are generally recorded and stored on computers at regular discrete time intervals. Strictly speaking, this is a special type of marked point process in which the points occur on a discrete set of regularly spaced times (e.g., daily values) rather than at any possible time. The occurrence of an exceedance in such cases can be modeled by using a discrete-time Markov chain (see section 5.2 of Lindsey, 2004).

\subsubsection{Choice of threshold}

The high threshold used to define the extreme events can be chosen in many different ways. The simplest approach is to choose a constant absolute threshold related to impacts; for example, the threshold of $25^{\circ} \mathrm{C}$ widely used to
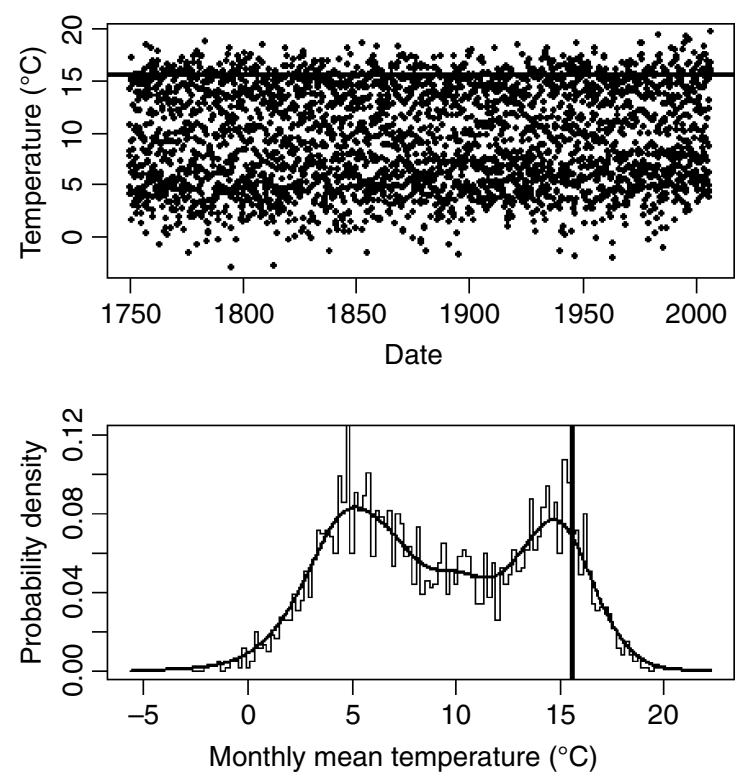

Figure 1.2. Monthly mean values of the central England temperature series from 1750 to 2006 (upper panel). Lower panel: the histogram of the monthly values ( $x$-axis, in ${ }^{\circ} \mathrm{C}$ ) together with a smooth kernel estimate of the probability density function. Ten percent of the values exceed the 90th quantile of $15.6^{\circ} \mathrm{C}$ (indicated by the solid line in both panels). 
define extreme heat wave indices (e.g., Alexander et al., 2006; New et al., 2006). Such extreme events can lead to severe health situations, which are likely to become more prevalent due to global warming (McGregor et al., 2005). A more relative approach is to choose a constant threshold based on the empirical distribution of the variable at each location; for example, the 90th quantile shown in the central England temperature example. This approach is useful in that it ensures that a given fraction (e.g., $10 \%$ ) of events will by definition be "extreme." In other words, it defines "extremeness" in terms of "rarity." In addition to these two approaches, one can also consider timevarying thresholds. For example, "record-breaking" events can be defined by choosing the threshold to be the maximum value of all previously observed values. One can also choose trending thresholds to help take account of nonstationarities such as the changing baseline caused by global warming. Such definitions of extreme events can help us avoid the paradoxical situation whereby "extreme events will become the norm" (as was stated by Deputy Prime Minister John Prescott after the autumn 2000 UK floods).

\subsubsection{Magnitude of the extreme events (distribution of the marks)}

The magnitude of the extreme events can most easily be summarized by calculating summary statistics of the sample of excesses; for example, the mean excess above the threshold (Coles, 2001). However, such an approach does not allow one to make inferences about as-yet-unobserved extreme values or provide probability estimates of extreme values that have reliable uncertainty estimates. It is therefore necessary to fit an appropriate tail probability distribution to the observed excesses (e.g., the sample of 308 excesses for the CET example).

Under rather general assumptions, a limit theorem shows that for most continuous random variables, the probability of exceedance above a large value $x>u$ is given by

$$
\operatorname{Pr}(X>x \mid X>u)=\left[1+\xi\left(\frac{x-u}{\sigma}\right)\right]^{-\frac{1}{\xi}}
$$

for a sufficiently high threshold $u$. This two-parameter distribution is known as the generalized Pareto distribution (GPD). The dimensional parameter, $\sigma$, defines the scale of the excesses, whereas the dimensionless parameter, $\xi$, defines the overall shape of the tail. These two parameters can be easily estimated by using maximum likelihood estimation.

For the CET example, the excesses above the 90 th quantile $u=15.6^{\circ} \mathrm{C}$ give a scale parameter estimate of $1.38^{\circ} \mathrm{C}$ (with a standard error of $0.09^{\circ} \mathrm{C}$ ) and a shape parameter estimate of -0.30 (with a standard error of 0.04 ). The 


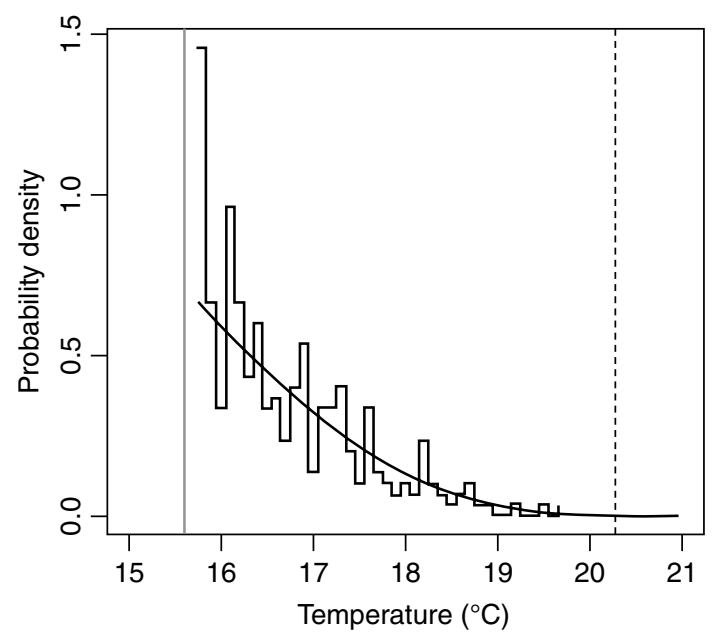

Figure 1.3. Histogram of the 308 monthly mean values of the central England temperature series from 1750 through 2006 that exceed the 90th quantile of $15.6^{\circ} \mathrm{C}$ (bold line) together with the GPD fit (continuous curve). The GPD fit has a finite upper limit at $20^{\circ} \mathrm{C}$, above which there is zero probability of a temperature value occurring.

negative shape parameter implies that the tail distribution has an upper limit at $u-\sigma / \xi=20.3^{\circ} \mathrm{C}$.

Figure 1.3 shows that the estimated GPD provides a good fit to the histogram of exceedance values. This is even clearer in the return level plot shown in Figure 1.4. The GPD fit can be used to estimate either the probability of exceedance above a chosen temperature (or its reciprocal, the return period), or the temperature quantile (the return level) corresponding to a specific exceedance probability. The GPD fit provides a smooth interpolation between the observed extreme values and a way of extrapolating beyond the maximum value observed in the finite sample.

\subsubsection{Timing of the extreme events (distribution of the points)}

There are two main approaches for estimating the rate of a point process: the counting specification, based on counting the number of points in fixed time intervals, and the interval specification, based on estimating the mean time interval between successive points (see Cox and Isham, 2000, p. 11).

The simplest counting specification approach involves dividing the time axis into a set of non-overlapping, equally spaced bins and then counting the number of points that fall into each bin. This approach gives rather noisy results due to the sharp bin edges. More efficient and smoother rate estimates 


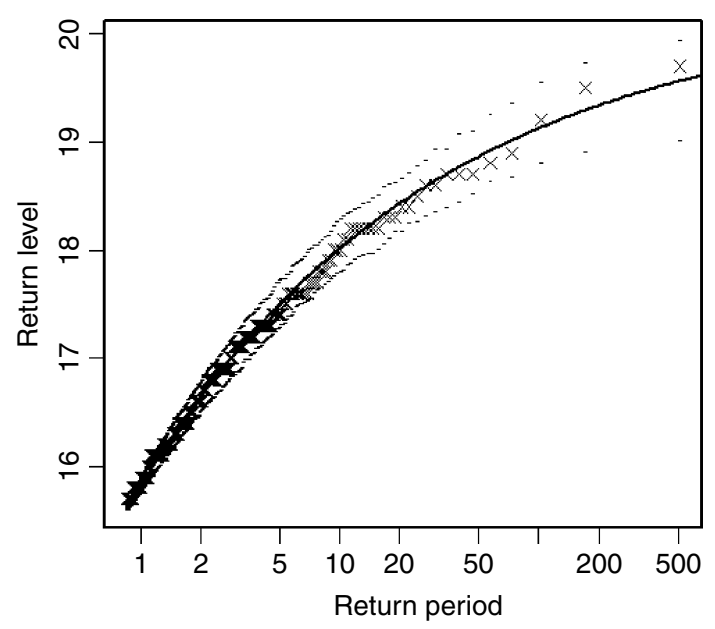

Figure 1.4. Return level plot for the central England temperature series from 1750 through 2006 . The return period is the reciprocal of the probability of a monthly temperature value exceeding the return level. The crosses denote the 308 empirical quantiles and probabilities, and the solid curve is the GPD fit. Note the concavity of the curve, which is characteristic of a tail distribution having a negative shape parameter.

can be obtained by using smooth local weighting based on a smooth kernel function rather than a sharp-edged bin (Diggle, 1985). Such an approach was recently used to investigate extreme flooding events in eastern Norway as observed in paleoclimatic lake sediments (Bøe et al., personal communication). Various tests can be used to test for trends in the rate of a Poisson process, but these have differing abilities to detect trends (Bain et al., 1985; Cohen and Sackrowitz, 1993).

In addition to characterizing changes in the rate and magnitude of a point process, one can also investigate the temporal clustering. For example, Mailier et al. (2006) used simple point process overdispersion ideas to evaluate the clustering in transits of extratropical cyclones. They found that there is significant clustering of cyclones over Western Europe that can be attributed to rates varying in time due to the dependence on large-scale flow patterns. In general, time dependence in rates for an extreme event can lead to clustering. This is a very interesting area for future research on extreme weather and climate events.

\subsubsection{Some ideas for future work}

Statistical analysis of extreme events generally focuses on a given set of extreme events and tends to neglect how such events came into existence. 
This is a strength in that it makes extreme value techniques more universally applicable to different areas of science no matter how the extremes formed. However, this approach also has a weakness in that it ignores information about the process that led to the extreme events that could help improve inference. How moderately large events evolve into extreme events can provide clues into the very nature of the extreme events. The dynamical knowledge about underlying formation processes should be exploited in the statistical analysis.

\subsection{The origin of extreme events}

Understanding the processes that lead to the creation of extreme events and how they might change in the future is a key goal of climate science. To help tackle the problem of the origin of extremes, I propose two guiding principles.

- The evolutionary principle. Extreme events do not arise spontaneously: instead, they evolve continuously from less extreme events and they stop evolving to become even more extreme events.

- The stationary principle. Extremes such as local maxima and minima are quasistationary states in which the rate of change of their amplitude is zero. This characteristic implies that there is an interesting balance between forcing and dissipation tendencies for such extreme events.

There are various processes that can give rise to extreme events:

- Rapid growth due to instabilities caused by positive feedbacks; for example, the rapid growth of storms due to convective and baroclinic instability.

- Displacement of a weather system into a new spatial location (e.g., a hurricane in Boston) or into a different time period (e.g., a late frost in spring).

- Simultaneous coincidence of several non-extreme conditions (e.g., freak waves caused by several waves occurring together).

- Localization of activity into intermittent regions (e.g., precipitation in intertropical convergence zones).

- Persistence or frequent recurrence of weather leading to chronic extremes as caused by slower variations in the climate system (e.g., surface boundary conditions).

- Natural stochastic/chaotic variation that will lead to more extreme values being recorded as the time length of the record increases.

Understanding these processes is the key to understanding how extreme events have behaved in the past and how they might behave in the future. In addition to being of interest because of their large impacts, extreme events are worth studying because they can reveal insights into key processes. For example, investigation of rapidly deepening Atlantic storms ("bombs") has helped improve scientific knowledge of fundamental baroclinic instability 
mechanisms (explosive cyclogenesis). For numerical weather and climate models to correctly simulate extreme events, they will need to adequately represent such processes.

\subsection{Conclusion}

This chapter has addressed the perplexing issues of how to define and diagnose extreme events. It has been shown that extreme events are generally complex entities described by several different attributes: rate of occurrence, magnitude (intensity), temporal duration and timing, spatial structure, and multivariate dependencies.

Despite this complexity, extreme weather and climate events are often described by using only a single variable (e.g., maximum wind speed at landfall). Exceedances of such a variable above a high threshold define what is known as simple extreme events. This simple description of complex events can be considered to be a realization of a stochastic marked point process. Point process techniques can be usefully employed to characterize properties of simple extreme events such as the rate, the magnitude, and temporal clustering.

Despite the societal relevance, estimates and predictions of extreme events are prone to large sampling uncertainty due to the inherent rarity of such events. Careful inference is needed to make definitive statements about extreme events such as the regional changes one is likely to see due to global warming (e.g., Beniston et al., 2006). Inference can be improved by various approaches such as extrapolating from less extreme events (e.g., using tail distributions such as the generalized Pareto distribution), by pooling extreme events over a spatial region to reduce rarity (e.g., tropical cyclones over all the tropics), and by relating changes in extremes to changes in mean and variance (Beniston and Stephenson, 2004; Ferro et al., 2006). Such approaches require careful statistical modeling that can benefit from insight gained from knowledge of dynamical processes that determine extreme events.

\section{Acknowledgments}

I thank Rick Murnane and Henry Diaz for inviting me to present these ideas in the opening seminar at the "Assessing, Modeling, and Monitoring the Impacts of Extreme Climate Events" workshop in Bermuda, October 13-14, 2005. Many of the ideas presented here have grown out of exciting discussions that I have had with colleagues over the past few years; in particular, with Dr Chris Ferro and Professor Martin Beniston. Finally, but not least, I thank the reviewers of this chapter for their useful comments. 


\section{References}

Alexander, L. V., Zhang, X., Peterson, T. C., et al. (2006). Global observed changes in daily climate extremes of temperature and precipitation. Journal of Geophysical Research (Atmospheres), 111, D05109, doi:10.1029/2005JD006290.

Bain, L. J., Engelhardt, M., and Wright, F. T. (1985). Tests for an increasing trend in the intensity of a Poisson process: a power study. Journal of the American Statistical Association, 80(390), 419-22.

Beniston, M., and Stephenson, D. B. (2004). Extreme climatic events and their evolution under changing climatic conditions. Global and Planetary Change, 44, 1-9.

Beniston, M, Stephenson, D. B., Christensen, O. B., et al. (2006). Future extreme events in European climate: an exploration of regional climate model projections. Climatic Change, PRUDENCE special issue.

Cohen, A., and Sackrowitz, H. B. (1993). Evaluating tests for increasing intensity of a Poisson process. Technometrics, 35(4), 446-8, doi:10.2307/1270277.

Coles, S. (2001). An Introduction to Statistical Modeling of Extreme Values. London: Springer-Verlag.

Cox, D. R., and Isham, V. (2000). Point Processes. New York: Chapman \& Hall/CRC.

Daley, D. J., and Vere-Jones, D. (2002). An Introduction to the Theory of Point Processes, 2nd edition. Berlin: Springer-Verlag.

Diggle, P. J. (1983). Statistical Analysis of Point Processes. London: Chapman \& Hall.

Diggle, P. J. (1985). A kernel method for smoothing point process data. Applied Statistics, 34, 138-7.

Ferro, C. A. T., Hannachi, A., and Stephenson, D. B. (2006). Simple non-parametric techniques for exploring changing probability distributions of weather. Journal of Climate, 18, 4344-54.

Intergovernmental Panel on Climate Change (IPCC) (2001). Climate Change 2001: Synthesis Report. Cambridge: Cambridge University Press.

Lindsey, J. K. (2004). Statistical Analysis of Stochastic Processes in Time. Cambridge: Cambridge University Press.

Mailier, P. J., Stephenson, D. B., Ferro, C. A. T., and Hodges, K. I. (2006). Serial clustering of extratropical cyclones. Monthly Weather Review, 134(8), 2224-40.

McGregor, G. R., Ferro, C. A. T., and Stephenson, D. B. (2005). Projected changes in extreme weather and climate events in Europe. In Extreme Weather and Climate Events and Public Health Responses, ed. W. Kirch, B. Menne, and R. Bertollini. Dresden: Springer, pp. 13-23.

New, M., Hewitson, B., Stephenson, D. B., et al. (2006). Evidence of trends in daily climate extremes over southern and west Africa. Journal of Geophysical Research (Atmospheres), 111, D14102, doi:10.1029/2005JD006289. 